\title{
Dental Hand for Rural Population: Teledentistry
}

\author{
${ }^{1}$ Ankita Jain, ${ }^{2}$ Dara John Bhaskar, ${ }^{3}$ Devanand Gupta, ${ }^{4}$ Chandan Agali, ${ }^{5}$ Vipul Gupta, ${ }^{6}$ Ruhi Mark
}

\begin{abstract}
Purpose: To know any alternate method for the provision of dental needs for the rural population.

Introduction: Teledentistry is an upcoming field in dentistry that combines telecommunication technology and dental care. It is an exciting field that has endless potential. Teledentistry increased patient access to dental care, improved quality of care and the cost effectiveness. Teledentistry is also useful in long-distance clinical training and continuing education, screening and dentistlaboratory communication.
\end{abstract}

Materials and methods: This review article is formulated based on the available literature online. A thorough search was made on the PubMed and other reliable sources and then this review is formed.

Conclusion: Teledentistry may provide a possible solution to many prevailing problems related to dental care provision, like people living in rural areas and those who are not able to retrieve regular dental care. The future of teledentistry will depend on the efforts of the health authorities as on the collective efforts of the dental professionals. Most of the dentists are unaware about teledentistry, about its goals and advantages and how to get involved into it.

Keywords: Teledentistry, Teleconsultation, Upcoming, Telecommunication.

How to cite this article: Jain A, Bhaskar DJ, Gupta D, Agali C, Gupta V, Mark R. Dental Hand for Rural Population: Teledentistry. J Contemp Dent 2014;4(1):27-29.

Source of support: Nil

Conflict of interest: None

\section{INTRODUCTION}

There are many barriers for the rural population to access specialty dental care, such as geographic remoteness, poor or no public transportation and poverty, leading to a compromise on quality health care, resulting in complications. ${ }^{1}$ Advances in dental care have documented that early diagnosis, preventive treatments, and early intervention can prevent or reduce the progress of most oral diseases, conditions that,

\footnotetext{
1,3,6 Postgraduate Student, ${ }^{2}$ Professor and Head

${ }^{4}$ Reader, ${ }^{5}$ Senior Lecturer

${ }^{1-5}$ Department of Public Health Dentistry, Teerthanker Mahaveer Dental College and Research Centre, Moradabad, Uttar Pradesh India

${ }^{6}$ Department of Periodontology, Teerthanker Mahaveer Dental College and Research Centre, Moradabad, Uttar Pradesh, India

Corresponding Author: Ankita Jain, Postgraduate Student Department of Public Health Dentistry, Teerthanker Mahaveer Dental College and Research Centre, Moradabad, Uttar Pradesh India, Phone: 09808723156, e-mail: ankitajain.0815@gmail.com
}

when left untreated, can have painful, disfiguring and lasting negative health consequences. ${ }^{2}$ Technologic innovations in the field of dentistry have been extensive in recent years. Most important advances have been made in the use of computers, telecommunication technology, digital diagnostic imaging services, devices and software for analysis and follow-up. ${ }^{3}$ Just as communication technology and uses of electronic information have developed over the years, terms to describe health care services at a distance, such as telehealth and telemedicine, have also evolved. Teledentistry is a combination of telecommunications and dentistry. 'Tele' is a Greek word meaning 'distance' and 'mederi' is a latin word meaning 'to heal'. ${ }^{4}$

Telemedicine (and by inclusion teledentistry) has been defined in a number of ways. One definition is: "the practice of healthcare delivery, diagnosis, consultation, treatment and education using interactive audio, video or data communications' (Golder and Brennan, 2000). ${ }^{5}$ Teledentistry is a rapidly forming subset of telehealth, a field that already has considerable impact on the health care industry. Cook in 1997 defined 'teledentistry' as 'the practice of using video-conferencing technologies to diagnose and provide advice about treatment over a distance' ${ }^{6}$ Teledentistry is an exciting new area of dentistry that uses electronic health records, telecommunications technology, digital imaging and the internet to link health care providers in rural or remote communities to enhance communication, the exchange of health information, and access to care for underserved patients, including socially disadvantaged people, those who live in remote or rural areas. ${ }^{2,7} \mathrm{New}$ information technology has not only improved the quality of management of dental patients but also has made possible their partial or complete management at distances of thousands of kilometers away from healthcare centers or qualified dentists. The entire process of networking, sharing digital information, distant consultations, workup, and analysis is dealt by 'teledentistry'. 8

Through the use of telecommunication and computer technologies, it is now possible to provide interactive access to specialist opinions that are not limited by the constraints of either space or time. The referring dentist logs into a secure web server and fills in the patient's details, the specific reasons for the consultation, the chief complaints and the provisional diagnosis information and attaches the digital intraoral images and the scanned digital dental radiographs. The specialist subsequently logs into the secure web server, 
reviews the case and suggests his diagnosis and treatment plan within a limited period. ${ }^{9}$

\section{MATERIALS AND METHODS}

We formulate a review article based on the available literature online. Data is collected from the search engine google. All the useful articles which we have included are easily accessible online. All the articles which are taken from web are properly quoted in the references. The useful information available in the references is systematically presented in this article. All the important information is compiled together and presented in the article.

\section{HISTORY OF TELEDENTISTRY}

The initial concept of teledentistry developed as part of the blueprint for dental informatics, a new domain combining computer and information science, engineering and technology in all areas of oral health, which was drafted at a 1989 conference funded by the Westinghouse electronics system group in Baltimore. ${ }^{1}$ Its focus was a discussion of how to apply dental informatics in dental practice. Teledentistry was put into practice in US army in 1994 by doing dental consultations on person located more than 100 miles apart. Since then, various institute and organization have practiced teledentistry with varying degree of success. In the 1990s, the concept of 'teledentistry' was introduced. Cook, in 1997, defined this as 'the practice of using video-conferencing technologies to diagnose and provide advice about treatment over a distance' (Cook J ISDN video conferencing in postgraduate dental education and orthodontic diagnosis). ${ }^{2-4}$

\section{METHODS OF TELECONSULTATION}

Teleconsultation through teledentistry can take place in either of the following ways: 'real-time consultation' and 'store-and forward method'. Real-time consultation involves a videoconference in which dental professionals and their patients, at different locations, may see, hear and communicate with one another. Store-and-forward method involves the exchange of clinical information and static images collected and stored by the dental practitioner, who forwards them for consultation and treatment planning. The third method has also been described, known as 'remote monitoring method', in which patients are monitored at a distance and can either be hospital-based or home-based. A 'near-real-time' consultation has also been mentioned in the literature, which involves low resolution, low frame rate product that looks like jittery television. patient is not present during the 'consultation'. Dentists can share patient information, radiographs, graphical representations of periodontal and hard tissues, therapies applied, lab results, tests, remarks, photographs and other information transportable through multiple providers. ${ }^{1,3,4,5}$

\section{BENEFITS OF TELEDENTISTRY}

- Reduced cost of service and improved quality of care.

- Reduction in peer isolation and increased specialist support as well as education.

- General dentists will send multimedia patient records to dental specialists, often enabling the specialist to make a diagnosis and develop a treatment plan without having to see the patient in person.

- Improvement in diagnostic services.

- Improved integration of dentistry into the larger health care delivery system.

- Improvement in communication with the insurance industry with respect to requirements.

- Improvement in communication with dental laboratories. ${ }^{4,6,7}$

\section{LEGAL ISSUES RELATED TO TELEDENTISTRY}

There is considerable variation between countries in terms of accountability, Licensure, jurisdiction, liability, privacy, consent and malpractice. The latter appears to be a major impediment to the use of teledentistry across borders. Teledentistry allow professionals to practice across broad geographic areas, some difficult ethical, legal and regulatory concerns are raised which point out that 'patients will need to be advised of the inherent risks of improper diagnosis and/or treatment due to failure of the technology involved'. Confidentiality patients should be made aware that their information is to be transmitted electronically and the possibility exists that the information will be intercepted, despite maximum efforts to maintain security. ${ }^{1,4,6,8,9}$

\section{CONCLUSION}

Currently, teledentistry has not yet become an integral part of mainstream oral health care. In the near future, teledentistry will be just another way to access an oral health care, especially encouraging for isolated population who may have difficulty accessing the oral health care system due to distance, inability to travel, or lack of oral health care providers in their area. Future advances in technology will enable teledentistry to be used in many more ways, such as clinical decision support, quality and safety assessment, consumer home use, medication e-prescribing and simulation training. Teledentistry provides new opportunities for dental education by providing the primary care professionals with an easy access to efficient consultation and by helping in conducting postgraduate education and continuing dental education programs. In spite of some issues which need to 
be resolved, the potential of teledentistry is tremendous, which needs to be explored.

\section{REFERENCES}

1. Reddy KV. Using teledentistry for providing the specialist access to rural Indians. Indian J Dent Res 2011;22:189.

2. Fricton J, Chen H. Using teledentistry to improve access to dental care for the underserved. Dent Clin North Am 2009;53:537-548.

3. Mihailovic B, Miladinovic M, Vujicic B. Telemedicine in Dentistry (Teledentistry), Advances in Telemedicine: Applications in Various Medical Disciplines and Geographical Regions. Graschew G (ed), 2011, ISBN: 978-953-307-161-9, InTech.

4. Sanjeev M, Garg SK. Teledentistry a new trend in oral health. Int J Clin Cases lnvest 2011;2:49-53.
5. Ata SO, Ozkan S. Information technology in oral health care: attitudes of dental professionals on the use of teledentistry in Turkey. Eur Mediterranean Conf Information System 2009;1-8.

6. Bhambal A, Saxena S, Balsaraf SV. Teledentistry: potentials unexplored. J Int Oral Health 2010;2:1-6.

7. Torres-Pereira CC, Morosini Ide A, Possebon RS, Giovanini AF, Bortoluzzi MC, Leão JC, Piazzetta CM. Teledentistry: distant diagnosis of oral disease using e-mails. Telemed JE Health 2013;19(2):117-121.

8. Chhabra N, Chhabra A, Jain AL, Kaur H, Bansal S. Role of teledentistry in dental education: need of the era. JCDR 2011; 5:1486-1488.

9. Jampani ND, Nutalapati R, Dontula BSK, Boyapati R. Applications of teledentistry: a literature review and update. J Int Soc Prev Comm Dent 2011;1:37-44. 